\title{
Los sistemas de información geográfica y los planes de ordenamiento territorial en Colombia ${ }^{1}$
}

The geographic information systems and land use plans in Colombia

\section{Resumen}

\section{Jahir Alexander Gutiérrez Ossa ${ }^{2}$ Gleidy Alexandra Urrego Estrada ${ }^{3}$}

Este artículo de reflexión está dirigido a analizar la aplicabilidad y el contexto de los sistemas de información geográfica (SIG), en el ordenamiento y la planeación territorial para el desarrollo en Colombia. La implementación de los sistemas obedece a la relevancia de codificar y monitorear por medio de las herramientas tecnológicas, la información que se tiene de la estructura geográfica de cada región, marco que implica la revisión de las formas estipuladas hasta ahora para evaluar y hacer gestión territorial. Mediante los sistemas, como guía y metodología, se espera superar los vacíos relacionados con la poca estructura de datos e información geográfica que se tiene al servicio de las administraciones públicas territoriales. Uno de los hallazgos cruciales derivados de dicha fundamentación radica en poder establecer los criterios de convergencia entre los planes de ordenamiento y los de desarrollo territorial, tan dispares en el país.

Palabras clave: Modelos de planificación, políticas de desarrollo regional, recopilación de datos, usos del suelo.

1 Este artículo es producto del proyecto de investigación Parámetros del sistema de información geográfica aplicados al ordenamiento territorial nacional (SIG-OTN) en la planeación municipal y el ordenamiento territorial en Antioquia, financiado por la ESAP (Escuela Superior de Administración Pública), en el período agosto de 2009 a diciembre de 2009, ejecutado por el grupo de investigación en Política, Derecho y Gestión Pública de la ESAP, categoría A en Colciencias.

2 Ph.D. en Administración Pública. Líder del Grupo en Gestión Empresarial, Facultad de Ciencias Administrativas y Económicas, Universidad CES. Medellín- Colombia. algutierrez@ces.edu.co; jagogutierreez@gmail.com

3 Administradora Pública. Grupo de investigación en Política, Derecho y Gestión Pública de la ESAP. Medellín- Colombia. gleidy. urrego@gmail.com 


\section{Abstract}

This article of reflection is directed to analyze the applicability and the context of the Geographic Information Systems (GIS), in the ordering and land use planning for development in Colombia. The implementation of the systems is focused on the relevance of encoding and monitoring, through the technological tools, the information of the geographic structure of each region that implies the review of the forms stipulated until now for evaluating and making territorial management. Through the systems, as a guide and with their methodology, we expect to overcome the gaps related to the small amount of data and geographic information that serve territorial public administrations. One of the crucial findings derived from this fundamentation, is being able to establish the criteria of convergence between the plans of ordering and those of territorial development, which are so different in the country.

Key words: Data summary, models of planning, policies of regional development, uses of the ground. 


\section{Introducción}

La consolidación de los sistemas de información geográfica como base de datos espaciales y territoriales, está concebida para ordenar el acervo de referencias geográficas disponible por ahora en formato análogo. Al respecto, el Estado colombiano asumió la aplicación de los sistemas de información en la geografía local y regional por medio del Sistema de Información Geográfica para el Ordenamiento Territorial (SIG-OTN), a cargo del Instituto Geográfico Agustín Codazzi (IGAC, 2006), para establecer los procesos de ordenamiento y planeación territorial mediante los sistemas (art. 49 de la Ley 152 de 1994).

Los sistemas de información están contemplados para elevar la calidad de los puntos de análisis y relaciones que distinguen al territorio. No obstante, gran parte de su acierto está sujeto al grado de asimilación de las herramientas a las que haya lugar, y de la elaboración de los marcos necesarios para comenzar a apreciar el campo aplicativo de la información geográfica derivada de los sistemas territoriales, que constituyen el escenario físico-espacial de las interacciones producidas, a la hora de evaluar las características del territorio como base de información.

Los sistemas de información tienen como referencia las condiciones territoriales entre quienes ejecutan los planes de desarrollo, al ser asumidos parámetros de coordinación estamental e institucional. Así, la generación de información que permanentemente surte el espacio físico-espacial, permite clarificar la composición mediática a la que han sido sometidos la planeación y los proyectos, desdibujados del orden lógico previsto por las condiciones espaciales y geográficas, que de manera directa constituyen la hoja de ruta requerida para evaluar los parámetros territoriales.

El interés por establecer los criterios de correlación entre las estipulaciones del ordenamiento con la planeación a partir de los sistemas de información apenas está comenzando. Aún se está en la etapa de traslado de la cartografía y los mapas análogos al sistema digital, asimismo, se están desarrollando algunos programas y pruebas piloto en distintas zonas del país para ir articulando la iniciativa a las condiciones territoriales, ejercicios en los que ha jugado un papel fundamental la percepción que tienen los habitantes en cuanto a la imagen geográfica del territorio.

El artículo está compuesto por los siguientes temas, a saber: 1 . Un análisis sobre el escenario de la geografía en la interacción del ordenamiento, la planeación y el territorio, 2. La identificación del sendero de aplicación e instrumentalización de los sistemas de información, y 3. La elaboración del marco de referencias de las condiciones territoriales que representan generalmente los sistemas de información geográfica, teniendo en cuenta como ejemplo, al departamento de Antioquia. La perspectiva trazada está dirigida a concebir la lógica sistémica del análisis territorial. 


\section{Geografía: interacción entre el ordenamiento, la planeación y el territorio}

La geografía ha recuperado el carácter de fundamento en los criterios de aplicación territorial, toda vez que ha renovado el análisis de los procesos de ordenamiento y planeación en su papel de estimador referencial. El componente extensivo al que ha sido conmensurada, le ha valido para alcanzar una interacción mucho más proactiva en las decisiones tomadas sobre la proyección de los ejercicios de intervención territorial, hacia la obtención de información geográfica diversificada, que emerge precisamente por la heterogeneidad de los registros territoriales. Gutiérrez y Sánchez lo explican así:

"El territorio, así entendido, es el resultado de los múltiples y diversos sentidos de los cuales dotan la materialidad quienes lo habitan, semantizándola mediante las construcciones socioculturales visibles en las prácticas sociales" (2009, p. 56).

En vista de ello, el rastreo de la información geográfica recupera el estado de balance que debe prevalecer entre las perspectivas de transformación territorial con las oportunidades que brinda a partir del contexto espacial y dimensional. Un hecho significativo de la interacción es que ha fortalecido el interés por evaluar las condiciones territoriales de manera ex ante de cualquier intervención. De allí, que hoy tenga lógica habilitar el barómetro del análisis geográfico para sustentar las diversas aplicaciones a que haya lugar.
Entre el ordenamiento y los sistemas existe una especie de relación elíptica que permite ondear de manera extendida las interacciones cometidas entre ambos, debido a la capacidad de información generada por cuenta de dicha elongación. Por ende, el ordenamiento es la pieza clave para intensificar el proceso de consecución de plataformas que permiten concebir el entramado necesario para constatar el ascenso de los sistemas de información y entender las movilidades y transformaciones que se instalan por cuenta de la planeación territorial y las derivaciones que provee.

La planeación representa el ejercicio materializado de la combinación efectiva entre el flujo de datos obtenidos por cuenta de los sistemas junto con la capacidad de análisis y evaluación ofrecido por el ordenamiento territorial. A medida que se afianza la interacción de ambos, el ordenamiento termina por afinar la planeación, y esta última ilustra de manera profusa al ordenamiento. En este sentido, para lograr la complementariedad de ambos escenarios, resulta relevante definir los elementos que provocan el cierre de la brecha existente entre los dos. Afirma Castro (2009) que, de igual manera, el POT y la descentralización no son procesos antagónicos; en realidad son inseparables. Señala que la discusión sobre estos temas quedó en el debate y la voluntad política, lo cual no ha trascendido, al punto de estar prácticamente congelado el debate. Según este autor, la coyuntura está en: 1 . El tema político y 2. En el tema normativo y de orden constitucional. 
El componente territorial es el encargado de tamizar las pretensiones aseveradas, en primer lugar, con respecto a los alcances y perspectivas que desde el ordenamiento son trazadas en virtud de los usos del suelo. En segundo lugar, con relación a la vinculación de la planeación arraigada en los diferentes ejercicios de interacción aplicados sobre el territorio, y en tercer lugar, por el papel de enlace que cumple entre las acepciones consideradas desde el ordenamiento y las capacidades que surtiría el sistema para actuar sobre la evidencia geográfica. Según Escallón:

Existe una concepción generalizada de que el territorio en sí mismo constituye un sujeto heterogéneo de difícil agregación cooperativa, en el que hay un estímulo tácito a las adiciones individuales, sin marcos globalizantes que resultan en un desarrollo de los datos inconexos y muchas veces descoordinado y por tanto, incoherente. Esta forma de abordar el proceso reflejada tanto en acciones institucionales como individuales, ha traído múltiples problemas, no sólo por el fomento del aislacionismo sino por la costosa duplicidad de esfuerzos que estas prácticas indefectiblemente acarrean. La experiencia de muchos países ha demostrado que esta concepción puede ser superada con creces, mediante estrategias que involucran acciones a nivel nacional, institucional e individual (2006, p. 113).
Frente al territorio aparecen simultáneamente conceptos similares a éste, bajo un marco de redes y representaciones colectivas proporcionadas por las interacciones de los elementos físicos y humanos. El espacio, el paisaje, la región geográfica y el sentido de lugar, proporcionan la materialización y configuración del territorio, y, a su vez, la especificidad que les da origen. La noción de territorio ha variado, transformándose de acuerdo con la percepción, la finalidad, los acuerdos e intereses y las posibilidades que brinda en concordancia con la relación dialéctica de su naturaleza. (Ver Figura 1).

La configuración del territorio se entiende en la medida en que son advertidos los aspectos y elementos que sustentan su composición geomorfológica, por cuanto responden ante una realidad ineludible, mostrada a través de la formalización de los (SIG) y sobre el empaquetamiento al que es dirigido el territorio. Con esta herramienta, la consulta técnica sobre la dimensión, el espacio y la territorialidad de las localidades y las regiones será la constante, y, claro está, elevará los niveles de discusión entre los agentes decisores de la acción local. Argumenta Bozzano:

El espacio, entonces puede ser pensado como una determinación constitutiva e inseparable de las cosas y los procesos físicos; como la condición de lo extenso en tanto en categoría analítica; como el conjunto indisociable del que participan, por un lado, cierta disposición de los objetos 
Figura 1. Relación territorio, espacio, paisaje, región geográfica y lugar.

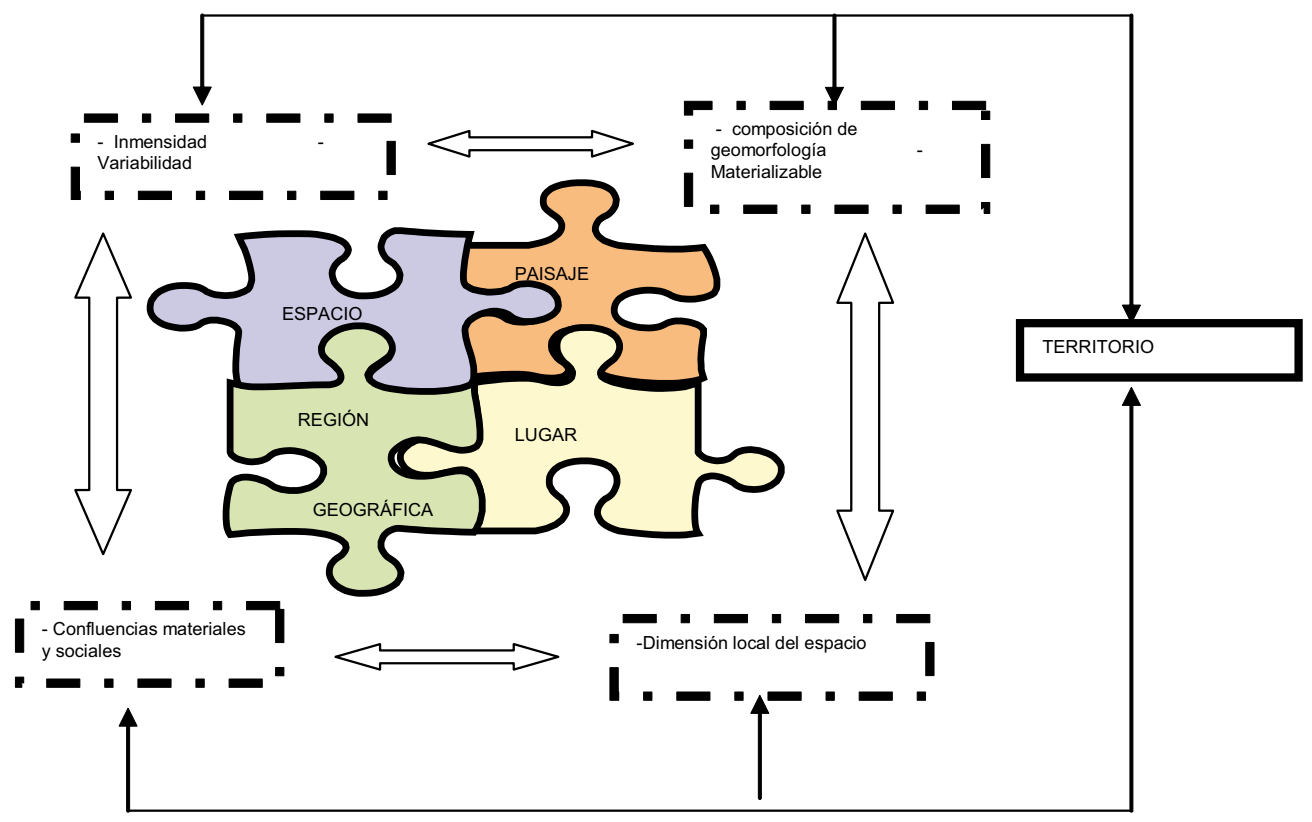

Fuente: elaboración propia.

geográficos, naturales y sociales, y por el otro, la vida que los llena y anima (2000, p. 25-26).

El territorio sigue siendo analizado desde una perspectiva enteramente de disposición, que no ha permitido profundizar en su estructura espacial y dimensional. Sin embargo, las variables que lo componen han tomado tal trascendencia, que son las que definen la forma en que es dispuesto el mismo. La concepción del territorio ha pasado por quienes consideran su relevancia como lugar, al igual que por quienes aprecian su importancia como escenario, siendo común ambas posturas al momento de congeniar los elementos en que debe abordarse la intervención territorial.
Es claro que los planes de desarrollo y los programas territoriales parten más de acuerdos subjetivos, afincados en los compromisos adquiridos en el quehacer de la acción política, y no propiamente por cuenta de una revisión exhaustiva y técnica de la vinculación de los componentes políticos a la revisión técnica de la estructura de análisis de los sistemas con trascendencia territorial. Por tal razón, temas como la capacidad de carga territorial y la concepción lógica del territorio, como asidero de información para la implementación de las propuestas políticas, no son complementarios. "El desarrollo territorial no se alcanza con la sumatoria de acciones sectoriales sino con la integración de conceptos y estrategias territoriales integradoras derivadas de la 
planificación regional, urbana rural y del desarrollo local" (DNP, 2010, p. 20).

El agravamiento al que ha sido conducida la intervención sobre el territorio, pese a la existencia de instituciones encargadas de velar por su gestión, no es congruente con el estado alterado en que se registra su disposición. En consecuencia, el ordenamiento territorial además de adquirir el carácter de instrumento corrector de las acometidas hechas en los planes de desarrollo, equilibra las alteraciones y transformaciones que sobre el uso del suelo han provocado las perspectivas que no han compatibilizado la vinculación territorial en ambos frentes.

\section{Aplicación e instrumentalización de los sistemas de información geográfica}

Los sistemas de información geográfica han servido para que temas como el ordenamiento y la planeación territorial constituyan, a partir de ellos, una base más sólida para definir el futuro de poblaciones y lugares, en cuanto a la distribución espacial de las personas, los recursos, la disposición de espacios geográficos, las estructuras y los distritos económicos y políticos, entre otros. De ahí se ha generalizado el tema del (SIG) en el ordenamiento territorial, denominado (SIG-OTN), sobre el que está toda la fundamentación e instrumentalización en el país.
El proyecto SIG-OTN busca conformar un sistema de información geográfica para la planificación y el ordenamiento territorial, que apoye de manera práctica los procesos de planificación y gestión del desarrollo con enfoque territorial y los actores del sistema nacional de planeación, en los ámbitos nacional, regional y local. Pretende aportar una herramienta técnica que soporte de manera eficiente y oportuna el proceso de toma de decisiones en la planificación, gestión y evaluación de políticas públicas, así como en la focalización y priorización de recursos de inversión con información político-administrativa, socioeconómica y ambiental georreferenciada (IGAC, 2008, p.7).

El enfoque territorial traza la dirección y la coordinación estratégica de las diversas áreas correspondientes a los deberes del gobierno. Se considera al SIG-OTN como herramienta informática para el mapeo, captura, análisis, manipulación, proceso y visualización de información espacial requerida en la planificación y ordenamiento territorial. La instrumentalización consiste en convertir la herramienta del SIG-OTN, más que en un proceso técnico, en elemento capaz de conducir a la administración pública para que tome las mejores decisiones frente a las condiciones del lugar en donde se desarrollan. De acuerdo con Gajardo et al. (2006, p. 82), actualmente se ha optado por la utilización de la geomática, la cual, básicamente, es la integración de múltiples tecnologías, como son los 
sistemas de información geográfica (SIG), la teledetección aérea y espacial, y los sistemas de posicionamiento global (GPS), como una herramienta para el manejo y gestión de bases de datos geográficos y territoriales. A pesar de las múltiples ventajas de procesamiento, consulta y almacenamiento de información que estos sistemas poseen, no son una novedad las limitaciones que presentan a la hora de realizar labores de análisis y modelación territorial. En este sentido, la respuesta y una posible solución a estas limitaciones pueden darse en la integración de métodos externos en el ámbito de la geomática, entre los que se encuentran las Técnicas de Evaluación Multicriterio (EMC).

Las herramientas tecnológicas implementadas por medio del SIG-OTN, permiten conocer la información geográfica desde la estructura espacial para el manejo de las cartas catastrales, los registros y las ubicaciones de predios, al igual que la definición y el límite de zonas destinadas para algún fin. Los SIG son la herramienta que fortalece el proceso de planeación y ordenamiento territorial, en la captura, análisis, manipulación, procesamiento y visualización de información espacialgeográfica, sobre la cual cada región se define e identifica. Su objetivo es "Contribuir a una eficiente y oportuna toma de decisiones, apoyando a los actoresautoridades e instancias en el sistema de planeación a nivel nacional, regional y local, con información político-administrativa, socio-económica y ambiental georreferenciada que soporte la gestión del desarrollo" (IGAC, 2009, p.5).
La instrumentalización tiene como premisa llevar la propuesta de una manera accesible a todos los entes obligados a comprender la magnitud de la iniciativa, para reorientar el esquema de trabajo de las secretarías y unidades adscritas a planeación en las localidades y regiones. Así, la discusión de los planes de ordenamiento, entre otros, dejará de ser un proceso anclado a las instancias políticas para tratarse desde una mirada técnica, ajena a las rivalidades de intereses, mas no impermeable a ellos, a la hora de concebir los planes o modificaciones de los mismos. $\mathrm{Al}$ respecto, señala de nuevo el IGAC:

El SIGOT (Sistema de Información Geográfica para la planeación y el Ordenamiento Territorial Nacional) es una herramienta cuyo objetivo central es contribuir a una eficiente y oportuna toma de decisiones, apoyando a los actores-autoridades e instancias en el sistema de planeación a nivel nacional, regional y local, con información político-administrativa, socio-económica y ambiental georreferenciada que soporte la gestión del desarrollo (IGAC, 2009, p. 5).

La sistematización de la planeación por medio de los (SIG), más que una novedad informática, es la manifestación de llevar a terrenos más técnicos y depurables tanto a la realización como al norte sobre el que se instituye el ordenamiento territorial. Responde al llamado que se hace para que ambos momentos territoriales correspondan a un balance entre las intenciones de quienes asumen la dirección 
territorial, municipal o departamental, y las dimensiones y connotaciones del lugar sobre el cual se desarrollan ambas propuestas (ver Figura 2).

Los sistemas de información logran destacarse plenamente al momento de superar la etapa de estimación y pronostico, por la del establecimiento de un orden de ideas que inciden en el mejoramiento de los manejos sobre los que termina expuesto el ordenamiento. Más que un patrón limitante y de ilustración técnica, debe tener la validez suficiente para difundir la necesidad de priorizar el disenso territorial bajo un orden lógico y pertinente conforme a los datos e información que emergen precisamente de las características provistas de las áreas geográficas consultadas. Explica el IGAC:
El proyecto SIG-OTN busca conformar un sistema de información geográfica para la planificación y el ordenamiento territorial, que apoye de manera práctica los procesos de planificación y gestión del desarrollo con enfoque territorial y los actores del sistema nacional de planeación, en los ámbitos nacional, regional y local. Pretende aportar una herramienta técnica que soporte de manera eficiente y oportuna el proceso de toma de decisiones en la planificación, gestión y evaluación de políticas públicas, así como en la focalización y priorización de recursos de inversión con información político-administrativa, socioeconómica y ambiental georreferenciada (IGAC, 2008, p.7).

Figura 2. POT frente al SIG-OTN.

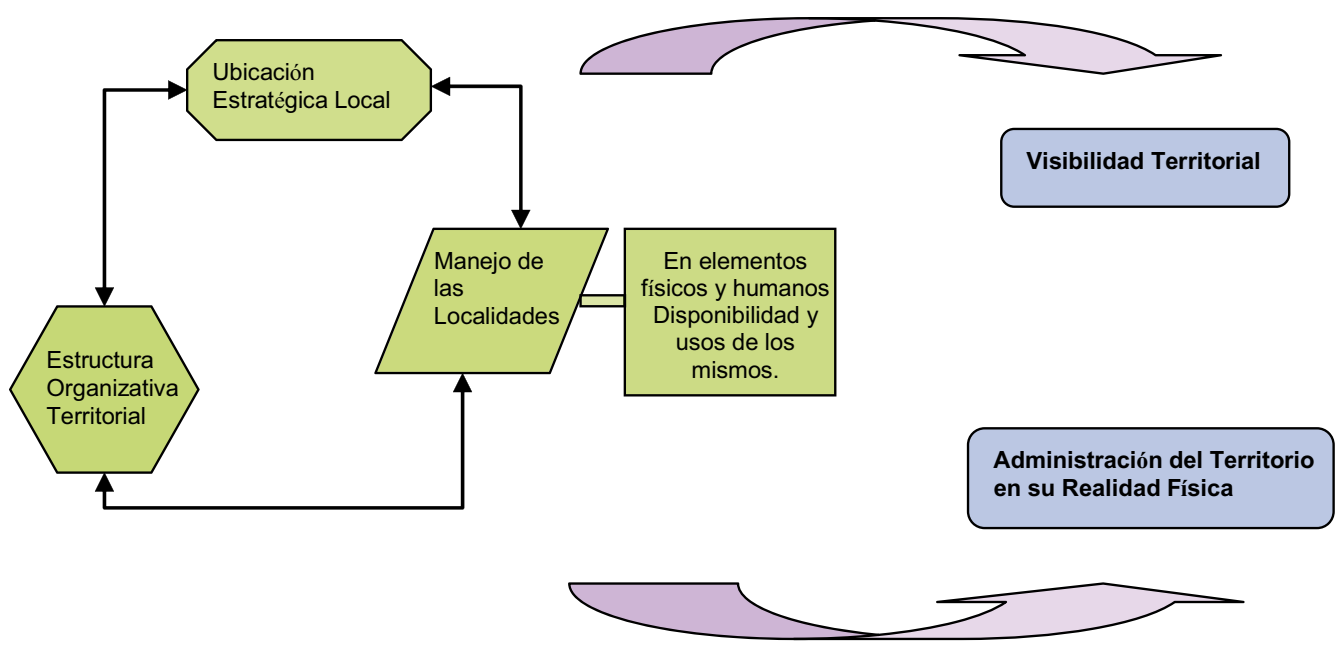

Fuente: elaboración propia. 
Es importante tener en cuenta que la progresividad de la articulación del SIGOTN, sólo será viable en la medida que se entienda la adaptación del sistema a la toma de decisiones y acciones sobre planeación y ordenamiento territorial, y esto será posible si hace parte de la técnica la necesidad de acogerla conforme a la ley pero aplicarla según sus ventajas, por parte de quienes finalmente toman las decisiones respecto a la misma, es decir, la dirigencia política, la administración municipal y las instituciones de orden nacional (ver Figura 3).

La importancia de estructurar un significado acorde a la utilización de los SIG es relevante, en la medida que permita ubicar al gobernante en el proceso planificador y de acción directa sobre la ordenación territorial, en correspondencia con las particularidades geográficas del territorio, que así como los planteamientos de sistemas, son también igualmente dinámicos, pero con indicadores menos controlables. Comúnmente el territorio tiene significados diversos que son cambiantes en los contextos en los cuales son analizados: "En el ámbito territorial se produce por la ocupación del suelo por parte de los elementos de generación, distribución y transporte, así como una explotación de recursos naturales" (Arancibia, 2000, p.2).

En el momento en que pueda combinarse el análisis de los SIG con el ordenamiento y la planeación territorial, se tendrá la capacidad suficiente para responder a temas neurálgicos relacionados con el cambio climático, la misma disposición de proyectos de infraestructura territorial, la capacidad de carga territorial, el tamaño y la dimensión que de manera igualmente física debe tener un territorio como prelación ante cualquier perspectiva espacial-geográfica o que con ellas traten de impulsar una nueva dimensión del concepto de localización o lugar. "Los SIG proporcionan el marco tanto para la comprensión de la dinámica territorial como para el desarrollo de acciones asociadas a su espacio de competencia" (Gómez, 2010, p. 172).

Es importante que pueda darse de manera técnica el espacio de discusión de los temas territoriales. Para ello es necesaria la capacitación en todos los escenarios, a fin de que sean las condiciones provistas por las herramientas técnicas a la par con las ilustraciones que proyectan o generan propuestas de cambio territorial, las que permitan de manera balanceada contemplar las posibilidades que mejor reparo tengan, sobre las condiciones en que se encuentran los territorios o hacia donde se espera llevarlos, de ahí que, de nuevo, se resalta la complementación entre el ordenamiento y la ejecución administrativa de la planeación.

\section{Sistemas de información geográfica sobre el ordenamiento y la planeación territorial en Colombia}

Colombia cuenta con 1.102 municipios, de los cuales entre el $90 \%$ y $95 \%$ se encuentran en las categorías $6^{\mathrm{a}}, 5^{\mathrm{a}}$ y $4^{\mathrm{a}}$ en el escalafón que al respecto señala la 
Figura 3. Modelo propuesto de SIG-OTN para el ordenamiento territorial municipal en Colombia.

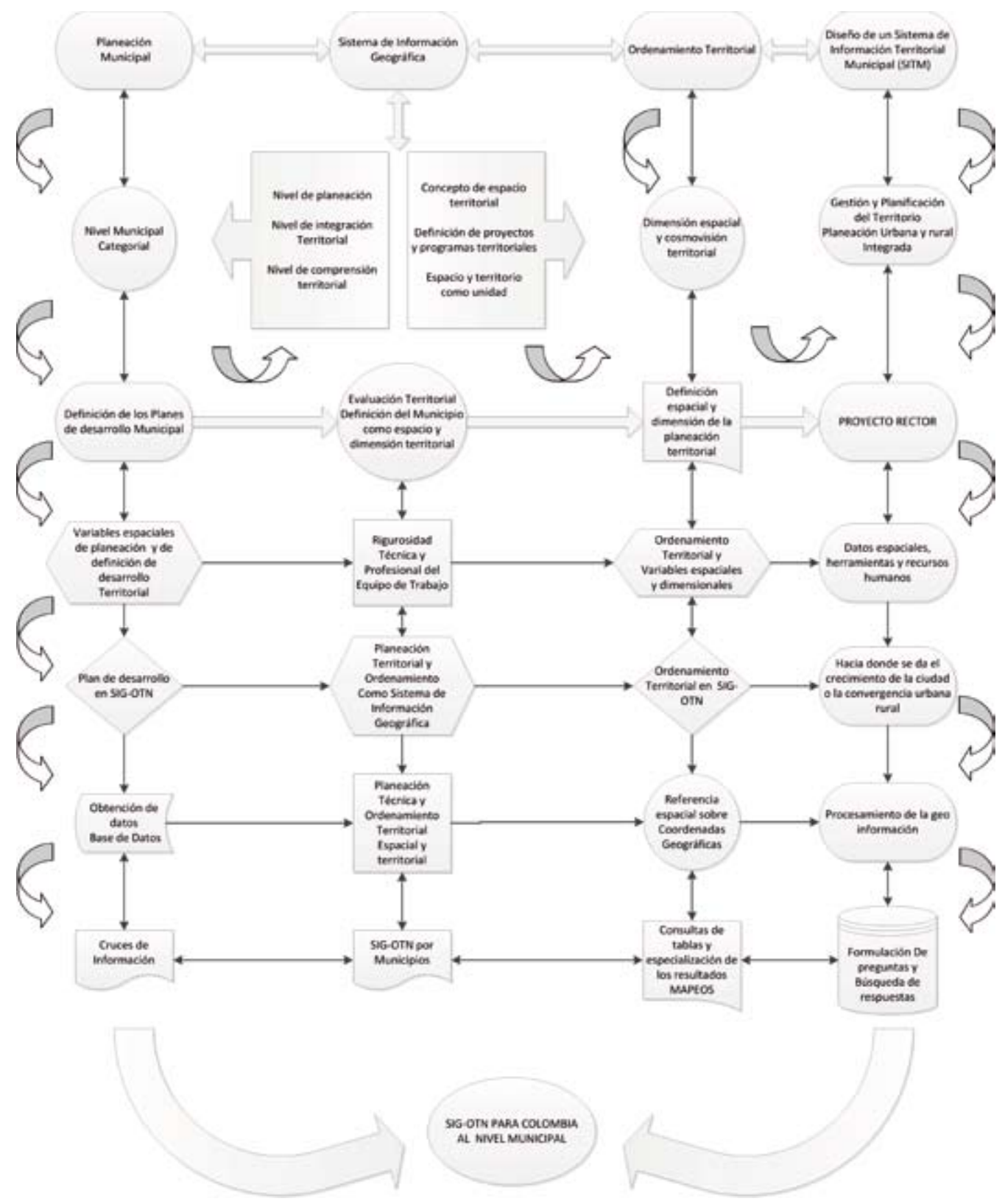

Fuente: elaboración propia a partir de IGAC (2008) 
Ley 617 de 2000, en el cual la categoría $6^{\mathrm{a}}$ equivale al $90 \%$. Dicha circunstancia implica afianzar la trascendencia que debe tener para ello la sistematización geográfica en aras de contribuir a pulir el escenario del ordenamiento y de planeación territorial que los reviste, puesto que en gran medida su ubicación administrativa tiene ascendencia en la poca mejora de su composición territorial, más que el aparente letargo institucional.

En la Constitución Política de 1991 se establecieron enfoques integradores pero con una exigua profundidad argumentativa en materia de ordenación del territorio. Aunque se presentaron ideas dominantes acerca de la autonomía y la ampliación de la descentralización de las entidades territoriales como eje central de dichas referencias, la tarea encomendada -de reglamentar las competencias específicas y coordinadas en un núcleo orientador bajo la Ley Orgánica de Ordenamiento Territorial- no se ha cumplido en las áreas biofísicas, demográficas, ecológicas y urbanísticas simultáneamente. Según Molina et al.: "La Ley de Ordenamiento Territorial establecida en la Constitución de 1991 es más una Ley de organización de competencias de los entes territoriales autorizados por la misma carta, que una Ley de acomodamiento, de ordenanza o de ordenamiento territorial de las mismas" (2004, p. 166).

La legislación Colombiana, por medio de la Ley 388 de 1997, ordena a los municipios y distritos del país la formulación de sus respectivos planes de ordenamiento territorial, para garantizar el desarrollo sostenible del territorio y el uso equitativo y racional del suelo. Además de integrar el marco legal vigente, así mismo, suministra un conjunto de herramientas que posibilitan alcanzar los objetivos propuestos a la luz del ordenamiento territorial. En adelante, la capacidad física de los territorios determinará la capacidad de gobernación de los municipios y las expectativas políticas. Al respecto argumenta Chaparro:

A pesar de los anteriores esfuerzos, aún no es posible cumplir con las exigencias de las entidades nacionales y territoriales para una efectiva formulación y ejecución de sus planes de desarrollo y de ordenamiento territorial. Situación que se origina en la insuficiente asignación de recursos presupuestales, y en la falta de coordinación interinstitucional e intersectorial, así como en la insuficiente capacidad instalada de las entidades productoras de información a nivel nacional para satisfacer las exigencias del mercado (2007, p. 24).

En diversos contextos ha sido señalada la ausente convergencia entre las estipulaciones de los planes de desarrollo con las previsiones, que fruto de su maduración aplicativa, ofrece el ordenamiento. Sin embargo, el factor mediador para que pueda elaborarse el marco lógico con el que tanto el vaciamiento de la información, como la postulación de las áreas enmarcadas para que sean una sola herramienta, dependen de los ejercicios de planeación que contemplen los criterios de la información territorial y de la 
estructura de datos, obtenida de forma espacial, física y geográfica, como se aprecia en el Decreto 2442 de 2006, con el cual se crea la Comisión Colombiana del Espacio, en el que se contempla el acuerdo número 3 del mismo decreto, a saber:

En las bases del Plan Nacional de Desarrollo 2006-2010 se plantea la conveniencia de que el Gobierno Nacional consolide una política espacial e implemente proyectos que utilicen las tecnologías espaciales en campos como la educación, la salud, la vigilancia ambiental, la gestión de recursos naturales, la atención de desastres, el monitoreo de los usos del suelo, las previsiones meteorológicas, el estudio de cambios climáticos, el transporte terrestre, la navegación marítima, fluvial y aérea y las telecomunicaciones. Que el Plan Nacional de Desarrollo establece así mismo que, a través de la Comisión Colombiana del Espacio, se formulará y coordinará la ejecución de una política Nacional para propiciar el desarrollo de tecnologías espaciales y su aplicación productiva en todos los campos (IGAC, 2007, p. 21).

Temas como los planes de ordenamiento, los planes básicos, el esquema de ordenamiento, la planeación estratégica y la misma planeación municipal, comenzarán a ser herramientas técnicas reales que tengan como preludio la convergencia territorial y ajuste de las propuestas conforme a la dirección y rutas que el camino de los sistemas así destaque. Sobre ellos, el papel de los cuerpos colegiados institucionales existentes en las diferentes formas territoriales adquieren relevancia en la medida que se internalice el ejercicio para la evaluación de las diferentes propuestas. "En Colombia el tema ha sido un asunto marginal que en lo nacional solo ha buscado proponer nuevas unidades de división territorial" (Borja \& Castell, 1996, p. 20).

El Instituto Geográfico Agustín Codazzi (IGAC) es la entidad nacional que lleva adelante la implementación del SIG-OTN para todo el país, con la participación y colaboración directa, claro está, de entidades como el Departamento Nacional de Planeación, el Ministerio de Vivienda y del Medio Ambiente, el Ministerio de Agricultura, el Observatorio Astronómico de Bogotá, junto con otras entidades tanto de carácter nacional como descentralizadas. Dicha iniciativa viene siendo auspiciada con recursos del gobierno suizo por medio de la Agencia para la Cooperación y el Desarrollo Suizo (ACSDE), cuya primera etapa de intervención fue realizada entre el año 2005 y finales del año 2008. Al respecto, expresa el IGAC:

La Ley 152 de 1994 -Ley Orgánica del Plan de Desarrollo-, en su artículo 49, establece dentro de las medidas de apoyo técnico a los procesos de planeación, la conformación de un sistema de información que permita elaborar diagnósticos y realizar labores de seguimiento y control de los planes de desarrollo por parte de 
las entidades nacionales y terr toriales de planeación. Además, desde el 2000 el país ha avanzado en la definición de una estrategia nacional para el desarrollo de la infraestructura colombiana de datos espaciales, ICDE, con la que se pretende ordenar la producción de los datos, facilitar su acceso y uso por parte de los diferentes sectores de la sociedad, controlando el ciclo de vida de la información desde su creación o levantamiento hasta la entrega al usuario final (IGAC, 2008, p.41).

En el escenario de revisión territorial y a escala regional, es tal vez el departamento de Antioquia el que más requiere una revisión completa y exhaustiva del SIG-OTN, no sólo por la cantidad de municipios que lo componen, sino por la diversidad de alteraciones y condiciones geográficas, espaciales y dimensionales que definen su estructura territorial. En estimativos claros, el departamento ha hecho esfuerzos dirigidos a la definición de la composición subregional, pero no ha abonado el camino para atender el estado de correlación que debe primar entre ellas:

Desde la promulgación de la Ley 388 de 1997 (Ley de Desarrollo Territorial), el Departamento Administrativo de Planeación de Antioquia ha venido realizando esfuerzos para que el contexto regional se incorpore como marco de referencia de los planes de ordenamiento territorial de los mu- nicipios, mediante la formulación de las directrices de ordenamiento territorial para el departamento. Para ello, en el año 2002 preparó un documento para la elaboración de directrices de ordenamiento territorial (DAP, 2002), que se complementó en el 2004 y en el cual se desarrolló de manera preliminar un marco conceptual y metodológico para abordar unos lineamientos de ordenación territorial (Gobernación de Antioquia, Alcaldía de Medellín \& Área Metropolitana, 2008, p.37).

El departamento de Antioquia ha emprendido el camino de internalizar dicho esquema en las nueve subregiones. Con este propósito ha dispuesto una caracterización de los mismos bajo la propuesta del LOTA (Lineamientos para la Ordenación Territorial del Departamento de Antioquia), cuya idea es poner en marcha las iniciativas de información geográfica para la toma de decisiones en la planeación territorial y el mismo ordenamiento. Se considera como el paralelo del ICDE o, por lo menos, la base instrumental para comenzar a planear los elementos del ordenamiento territorial desde la aplicación de dicha infraestructura. Londoño manifiesta lo siguiente:

El Sistema Nacional para el Ordenamiento Territorial contenido en la Ley 388 de 1997 quedó sin marco departamental y subregional vinculante por la declaratoria de inexequibilidad del artículo 7, que definía las funciones de ordenamiento territorial de los departamentos mediante 
la elaboración de directrices departamentales, como marco territorial para el ordenamiento de los municipios de los departamentos. El Sistema de Planificación del Desarrollo regional por la Ley 152 de 1994 no se articula eficientemente con los procesos de ordenamiento territorial, dando como resultado que en el territorio departamental, la planificación del desarrollo no tenga expresión territorial vinculante y la planeación del desarrollo municipal a corto plazo, que encontrará planes de ordenamiento territorial de largo plazo ya adoptados o en revisión, no logrará la articulación necesaria para que todo el proceso sea integral (Londoño, 2008, p.119).

El rastreo inicial, hecho en la primera indagación sobre SIG-OTN en la planeación y el ordenamiento territorial en Antioquia, ha concluido recientemente, y ahora se encuentra en una segunda etapa, en la que se esperan consolidar los datos de información a escala territorial, al servicio de las administraciones públicas territoriales, para que sirva de eje y filtro transversal de la planeación, el ordenamiento y la proyección territorial. Por ende, el análisis debe centrarse en postular los horizontes que plantea dicha herramienta para cada nivel. Sobre el tema, anota Londoño:

El sistema para la planeación del desarrollo y el ordenamiento territorial en Colombia (Ley 152 de 1994 y Ley 388 de 1997) está incompleto debido a que faltan instrumentos vinculantes para el ordenamiento en los ámbitos nacional, regional y departamental. El ámbito departamental no cuenta con un instrumento jurídico vinculante que oriente la formulación de los planes de desarrollo y ordenamiento territorial de los municipios (Londoño, 2008, p. 126).

Los resultados que se esperan de los ejercicios de planeación, se evaluarán en la medida en que cada uno de ellos esté en sintonía con el otro, respondiendo en primera instancia, a las condiciones del territorio como una exigencia sin excepción; igualmente, dependerá de que ellos traduzcan bajo la elaboración del plan, las acciones que deben acometerse respecto a la intervención territorial; lectura que manifiesta la claridad conceptual e interpretativa de los frentes temáticos a apremiar con cada ejercicio, como se indica en seguida:

La clasificación jerárquica de los centros urbanos antioqueños permite observar las ciudades que tienen un mayor grado de importancia dentro de una región o dentro de un grupo de ciudades del departamento. Los centros urbanos clasificados en los niveles superiores corresponden a las ciudades más destacadas de Antioquia, en las cuales se desarrollan variedad de actividades comerciales, industriales, de transporte y de servicios; éstas también cuentan con equipamientos diversificados en salud, educación, entidades financieras, notarias, recreación y cultura. El nivel jerárquico de 
los centros urbanos va disminuyendo, en la medida en que los bienes y servicios que se ofrecen a la población son más escasos y los equipamientos son menos especializados (IGAC, IDEA \& Gobernación de Antioquia, 2002, p. 263).

El número de megaproyectos que se han puesto en marcha y los próximos a iniciar en cada una de las subregiones del departamento, obligan de inmediato a dar luces y caminos en la interpretación, aplicación y aporte de dichos estadios, pues la fuerza de las transformaciones territoriales está dejando por fuera del juego al ordenamiento y la planeación como elementos que, supuestamente, deben ser los guías rectores para atender estas realidades que se avecinan en la hoja de ruta de quienes administran lo territorial. En este punto vale la pena anotar lo expresado por la Gobernación y otras entidades de Antioquia:

El Sistema Departamental de Planificación-SPD- es un instrumento normativo para implementar un sistema de planificación articulado entre el departamento de Antioquia y sus municipios. Propone como referentes estructurales los lineamientos departamentales de ordenación territorial y las propuestas derivadas del Plan Estratégico de Antioquia -PLANEA-, promueve el fortalecimiento de las dinámicas regionales y armoniza estructuras institucionales con el fin de articular planes, programas y proyectos para ser aplicados en el proceso de planificación territorial del departamento de Antioquia. (Gobernación, Alcaldía \& Área Metropolitana, 2008, p. 377).

Los aplicativos derivados de los sistemas de información tendrán trascendencia en la medida que las estipulaciones y programaciones a que haya lugar en materia de intervención territorial partan del análisis lógico en el que estaría definida la información espacial y geográfica del territorio a considerar. Si bien el tema convoca a una revisión de la forma un tanto ajena con que se hace planeación, la estructura de datos que se obtendría con dichos aplicativos, además de estos, justificarían dicha revisión.

\section{Conclusiones}

La vigencia de los sistemas de información geográfica provocará un cambio directo en la Ley 617 de 2000, con respecto a la categorización territorial tanto de los departamentos como de los municipios. La etapa nominal de categorización atenderá los componentes dinámicos que constituyen cada una de las localidades y regiones del país. Lo interesante es que, a partir de los datos obtenidos, podrá mejorar la calidad en la administración de los recursos girados a las distintas entidades, $y$, de paso, se definirá un esquema de correspondencia geográfica y física que permita armonizar el estado territorial con la disposición presupuestal. 
Es deber de los cuerpos colegiados municipales y departamentales comenzar a preparar sus iniciativas y propuestas con un alto grado de análisis geográfico y con el reconocimiento del impacto de las consideraciones que conforme a las prerrogativas territoriales tengan participación directa en dicho escenario. Un buen principio de la administración pública es que quienes, en cualquier rango, desempeñen funciones públicas, tengan en cuenta en sus decisiones el impacto que debe guiar geográficamente cualquier acción sobre el espacio-territorial.

Por su parte, los diversos agentes y sujetos dispondrán de una herramienta excepcional para desarrollar los programas y proyectos que tengan consecuencia en los diferentes aspectos territoriales. Las áreas relacionadas con la intervención local y regional serán las que tengan más elementos de fondo para establecer un esquema claro de referencia entre lo propuesto y el impacto territorial.

El cambio al que definitivamente conducirá el desarrollo de la programación territorial conforme a los sistemas de información geográfica, mejorará el lenguaje en que se presentan los casos a revisión por parte de las instituciones públicas del orden nacional, departamental y municipal, pues si bien, en principio, tendrá un componente altamente técnico, este podrá ser planteado de manera que los involucrados con cada rubro no terminen la discusión por falta de fundamentos sino que permitan el acogimiento de las propuestas de forma directa en atención a las evaluaciones hechas desde la herramienta.

La consulta a la dirección y manejo territorial no será político-electoral sino político-técnica. En otras palabras, deberá existir una correspondencia entre los preceptos y percepciones propias del hecho político con la capacidad del territorio para responder ante lo que se le manifiesta con cada programa y proyecto incluido en el plan de desarrollo, en cuanto a la interpretación del territorio, aunque no desde la dimensión territorial. La geografía toma relevancia, por cuanto sobre ella es que en adelante se depositará toda la capacidad de acción pública y política del territorio. 


\section{Literatura citada}

Arancibia, E. (2008). El uso de los sistemas de información geográfica SIG en la planificación estratégica de los recursos naturales. Santiago de Chile: Universidad Bolivariana.

Borja, J. \& Castells, M. (1997). Lo local y lo global. Madrid, España: Taurus.

Bozzano, H. (2000). Territorios reales, territorios pensados, territorios posibles. Aportes para una teoría territorial del ambiente. Buenos Aires, Argentina: Espacio.

Castro, J. (2009,12 de enero). El nuevo municipio colombiano. [Programa televisado]. Colombia: Canal del Congreso de la República.

Chaparro, M. I. (2007). Pasos firmes para la consolidación de la infraestructura colombiana de datos espaciales. Semana Geomática (2006). Revista del Instituto Geográfico Agustín Codazzi IGAC (36), 20-26.

Departamento Administrativo de Planeación de Antioquia DAP. (2008). Lineamientos para el ordenamiento territorial en Antioquia LOTA. Medellín: Comisión Tripartita.

Departamento Nacional de Planeación DNP (2010). Orientaciones conceptuales y metodológicas para la formulación de visiones de desarrollo territorial. Bogotá, Colombia.

Escallón, J. O. (2006). Hacia una forma más eficiente de trabajar con información del territorio utilizando herramientas de captura, visibilidad y descubrimiento. Semana de Geomática (2005). Revista Análisis Geográfico (30), 112-120.

Gajardo, J., Mena, C. \& Ormazábal, Y. (2006). Modelación espacial mediante geomática y evaluación multicriterio para la ordenación territorial. Rev. Fac. Ing.-Univ. Tarapacá 14 (1), 81-89.

Gobernación de Antioquia, Alcaldía de Medellín y Área Metropolitana, Gobierno de Chile, Cooperación Técnica GTZ de Alemania (2008). Lineamientos de ordenación territorial para Antioquia. Medellín: Multigráficas.

Gómez, L. M. (2010). Tecnología SIG: gestión de la información como aporte al desarrollo del país. En IGAC-ICDE (2010). Sistema de Información Geográfica. Análisis Geográficos 46, 171-187. Bogotá: Imprenta Nacional.

Gutiérrez, A. L. \& Sánchez, L. M. (2009). Planeación para el desarrollo del territorio: perspectiva contemporánea. Grupo de Investigación Medio Ambiente y Sociedad del Centro. Medellín: Universidad de Antioquia.

Instituto Geográfico Agustín Codazzi IGAC. (2009). Manual SIG-OT en Colombia. Bogotá. Recuperado de https://sigotn.igac.gov.co/sigotn.

Instituto Geográfico Agustín Codazzi IGAC. (2008). Ordenamiento territorial, métodos de modelamiento y análisis espacial. Revista Análisis Geográficos (39), 1-176.

Instituto Geográfico Agustín Codazzi IGAC, Instituto para el Desarrollo de Antioquia IDEA \& Gobernación de Antioquia (2007). Antioquia. Características geográficas. Bogotá: Imprenta Nacional. 
Instituto Geográfico Agustín Codazzi IGAC. (2007). Semana Geomática. Revista Análisis Geográficos (38), 1-140.

Instituto Geográfico Agustín Codazzi IGAC. (2007). Análisis geográficos. Comisión Colombiana del Espacio I. Marco regulatorio y proyección de la tecnología espacial en Colombia. Semana Geomática. Revista Análisis Geográficos (35), 1-120.

Instituto Geográfico Agustín Codazzi IGAC. (2006). Análisis geográficos. Sistemas de Información Geográfica. Semana Geomática (2005). Revista Análisis Geográficos (32), 1-170.

Instituto Geográfico Agustín Codazzi IGAC \& Agencia Sueca para el Desarrollo Internacional ASDI. (2006). Proyecto desarrollo conceptual y metodológico de un sistema de información geográfica para el ordenamiento territorial nacional. Balance de actividades y resultados en 2006-Documento ejecutivo- Equipo local SIG-OT IGAC/ SWEDESURVEY.

Instituto Geográfico Agustín Codazzi IGAC. (2005). Revista Análisis Geográficos, Edicion especial (29).

Londoño, F. J. (2008). Proceso de formulación de los lineamientos para el ordenamiento territorial de Antioquia. En Instituto Geográfico Agustín Codazzi IGAC. Revista Análisis Geográfico (39).

Molina, C. M. et al. (2004). Organización del territorio: teorías, enfoques y tendencias. Centro de Investigaciones Socio-Jurídicas. Bogotá: Universidad Libre.

Plan Estrategico de Antioquia PLANEA. (2005). Atlas geoestratégico de Antioquia. Medellín: Instituto Geográfico Agustín Codazzi IGAC, Gobernación de Antioquia.

Recepción: 20 de agosto de 2011

Evaluación: 30 de agosto de 2011

Aprobación: 02 de septiembre de 2011 
